\section{PEDIATRIC REHABILITATION}

\author{
Poster 458 \\ Improvement of Premorbid Developmental Delay \\ Following Treatment of Post Traumatic \\ Hydrocephalus: A Case Report
}

Cristina M. Sanders, DO (University of Michigan, Ann Arbor, Michigan, United States), Joseph E. Hornyak IV, MD, PhD, Lisa M. Voss, DO

Disclosures: Cristina Sanders: I Have No Relevant Financial Relationships To Disclose

Case/Program Description: Patient is a 2-year-old male with a past medical history of Shone's complex, mild global delays and chronic hydrocephalus ex vacuo. Patient was admitted to outside children's hospital following fall from a bunk bed, approximately four feet. Initially, patient had reduced consciousness, vomiting, inability to maintain eye contact, and ataxic gait. He was found to have a small subdural hematoma, and unchanged hydrocephalus. Patient was monitored at outside hospital with neurologic improvement. Following the administration of midazolam for repeat imaging, patient became bradycardic and difficult to arouse. He was then transferred to our children's hospital for higher level of care. On PM\&R consult, he was found to have right esotropia. Given new neurologic finding, neurosurgery was asked to re-evaluate, and neuro-ophthalmology were consulted. Fundoscopic examination revealed papilledema, and rapid MR with increased hydrocephalus. ICP monitoring revealed significant intracranial pressure. Ventriculoperitoneal shunt was placed. Following shunt placement, esotropia was resolved. Patient returned to baseline mental status.

Setting: Large Tertiatry Pediatric Hospital.

Results: On outpatient follow up visit, to include imaging, patient's ventricular size was markedly reduced. Gait was with improved stability and speech was with improved vocabulary.

Discussion: While it was initially thought that patient's decline in function was attributed to post-traumatic hydrocephalus, improvements in imaging and pre-morbid function at follow up suggest pre-morbid hydrocephalus was pathogenic.

Conclusions: Patients with global developmental delay whom also present with chronic hydrocephalus ex vacuo may warrant more aggressive evaluation and management.

Level of Evidence: Level $\mathrm{V}$

\section{Poster 459}

Missed on Initial Imaging-Rare Head and Neck Injury: A Case Report

Michael K. Wempe, MD (University of Minnesota, Minneapolis, MN, United States), Angela Sinner, DO

Disclosures: Michael Wempe: I Have No Relevant Financial Relationships To Disclose

Case/Program Description: A 7-year-old male restrained passenger involved in a head-on motor vehicle collision was transported to an outside hospital where CT showed subarachnoid hemorrhages in the suprasellar cistern and anterior to the brainstem. CT Angiography Neck and CT Cervical Spine did not reveal any abnormalities. MRI Cervical spine showed edema in the posterior paraspinal muscles. He transferred from the outside hospital to our pediatric acute rehabilitation unit 17 days after his head-on motor vehicle collision for rehabilitation following a traumatic brain injury (TBI). On arrival, the patient had dysarthria, dysphagia, bladder incontinence, and bowel incontinence. He had weakness in all extremities. Upper extremities were more affected than lower extremities, and right extremities were more affected than left extremities. He had left abducens nerve palsy and left facial nerve paresis. With protrusion, his tongue deviated left. Setting: Pediatric Acute Inpatient Rehabilitation Unit.
Results: During a modified barium swallow, the patient was noted to have abnormal alignment of the cervical spine. Immediately, his case was discussed with Orthopaedic Spine Surgery and Neurosurgery, and these teams were formally consulted. The patient had atlantooccipital dislocation (AOD). He was placed in a cervical collar and then underwent an occiput to $\mathrm{C} 2$ posterior instrumentation and arthrodesis. Discussion: AOD was once considered lethal, and in the 1970 s, $6 \%-8 \%$ of all traffic fatalities had this injury. AOD is highly unstable and associated with brainstem injury, upper cervical cord injury, and cranial nerve injuries (VI, IX, X, XI, XII). This injury is frequently missed, and delayed diagnoses are common. CT and X-rays can look normal.

Conclusions: As it is frequently missed, one must have a high degree of suspicion to identify cases of AOD. Delayed identification can result in "catastrophic neurologic deterioration." Our patient had minimal cognitive findings and other deficits not consistent with TBI, raising the question of a missed injury.

Level of Evidence: Level $\mathrm{V}$

\section{Poster 461}

Recovery in Acute Inpatient Rehabilitation for Pediatric Anti-N-methyl-D-aspartate Receptor Encephalitis: A Case Report

Jacob Peacock, MD (Rusk Institute of Rehab Medicine, NY, New York, United States), Idris Amin, MD, Syed Ahmed, DO

Disclosures: Jacob Peacock: I Have No Relevant Financial Relationships To Disclose

Case/Program Description: A previously healthy 17-year-old female presented to Acute Pediatric Rehabilitation after diagnosis of Anti- $\mathrm{N}$ methyl-D-aspartate receptor encephalitis, a rare autoimmune disorder often associated with ovarian teratomas. The patient experienced a typical constellation of neurologic symptoms including psychosis, seizures, and progression to autonomic dysfunction, ataxia, paresis, and catatonia. The patient was enrolled in aggressive therapy in the inpatient setting after diagnosis and medical treatment in order to improve the physical and cognitive sequelae of the disorder.

Setting: Acute Pediatric Inpatient Rehabilitation.

Results: This patient initially presented with acute psychosis which progressed to unresponsive catatonia while in an inpatient psychiatric unit after a prodromal flu-like illness. After an initial work up to explore psychological, intracranial and infectious etiologies, an LP showed pleocytosis with lymphocyte predominance. The patient was started on acyclovir for viral encephalitis with dystonic storming. After seizing with worsened neurologic status, the patient was found to be NMDA-receptor Ab serum positive, and a grade II ovarian teratoma was found and emergently removed. After surgery, plasmapheresis, IVIG, and rituximab, the patient presented to acute rehab with significantly limited function after a 3-month hospital course. Initially, the patient was unable to vocalize and required assistance for ADLs due to cognitive deficits, poor endurance, and weakness. The AIR hospitalization focused on an interdisciplinary approach, including psychological support for the patient's family. After a 3-week course, the patient improved to supervision level for mobility, ADLs and IADLs. Cognitive function and speech significantly improved by the time of discharge, allowing the patient to safely return home under parental supervision.

Discussion: Interdisciplinary resources available in an acute rehabilitation setting allowed this patient to improve significantly in both function and safety at the time of discharge. The family significantly benefitted from coordination of care in order to transition home. The rehabilitation team's ability to utilize broad therapeutic methods assisted this patient to progress to a safe discharge.

Conclusions: An acute inpatient rehabilitation course can be of benefit to patients and families after severe neurologic decline due to Anti-NMDA encephalitis, as individuals often have significant deficits 